\title{
FUZZY DISTANCE AND FUZZY NORM
}

\author{
Masamichi Kon \\ Hirosaki University
}

(Received February 16, 2016; Revised July 29, 2016)

\begin{abstract}
We consider fuzzy sets on a metric, vector, or normed space. It is not assumed that the fuzzy sets have compact supports. In the present paper, a fuzzy distance and a fuzzy norm are proposed in order to measure the difference between two fuzzy sets, and their fundamental properties are investigated. Their definitions are based on Zadeh's extension principle. Although they are different from the classical ones based on the Hausdorff metric, they are suitable for data containing uncertainty or vagueness. The obtained results can be expected to be useful for analyzing such data when the data are represented as fuzzy sets.
\end{abstract}

Keywords: Fuzzy set, fuzzy distance, fuzzy norm

\section{Introduction}

The concept of fuzzy sets has been primarily introduced for representing sets containing uncertainty or vagueness by Zadeh [17] as fuzzy set theory. Then, fuzzy set theory has been applied in various areas such as economics, management science, engineering, optimization theory, operations research, etc. [4, 7, 13-16]. Zadeh's extension principle $[2,6,11,13,17]$, which provides a natural way for extending the domain of a mapping, is an important tool in the development of fuzzy arithmetic and other areas. For a mapping, fuzzy sets obtained by Zadeh's extension principle are images of other fuzzy sets on the domain of the mapping under the mapping. Some relationships between images of level sets of one or two fuzzy sets under a mapping and another fuzzy set obtained from the one or two fuzzy sets by Zadeh's extension principle are derived in [11]. Recently, they are extended to more general ones, and some useful results for applications are derived in [6]. In [6], it is suggested that a fuzzy distance and a fuzzy norm defined by Zadeh's extension principle are important and useful for applications. Although their definitions are different from the classical ones based on the Hausdorff metric [1], they are suitable for data containing uncertainty or vagueness. Because the classical fuzzy distance and fuzzy norm are real-valued, while our fuzzy distance and fuzzy norm are fuzzy set-valued.

In the present paper, the fuzzy distance and the fuzzy norm are defined by Zadeh's extension principle in order to measure the difference between two fuzzy sets, and their fundamental properties are investigated.

The remainder of the present paper is organized as follows. In Section 2, some notations are presented. In Section 3, a distance and a norm are defined for crisp sets, and their fundamental properties are investigated in order to investigate fundamental properties of the fuzzy distance and the fuzzy norm in Section 4. In Section 4, the fuzzy distance and the fuzzy norm are proposed, and their fundamental properties are investigated. In Section 5, some auxiliary results referred to in Section 4 are presented. Finally, conclusions are presented in Section 6. 


\section{Preliminaries}

In this section, some notations are presented.

Let $\mathbb{R}$ and $\mathbb{C}$ be sets of all real and complex numbers, respectively. We set $\mathbb{R}_{+}=\{x \in$ $\mathbb{R}: x \geq 0\}$ and $\mathbb{R}_{-}=\{x \in \mathbb{R}: x \leq 0\}$. Let $\operatorname{int}\left(\mathbb{R}_{+}\right)$and $\operatorname{int}\left(\mathbb{R}_{-}\right)$be interiors of $\mathbb{R}_{+}$and $\mathbb{R}_{-}$, respectively. For $a, b \in \mathbb{R}$, we set $[a, b]=\{x \in \mathbb{R}: a \leq x \leq b\},[a, b[=\{x \in \mathbb{R}: a \leq x<b\}$, ]$a, b]=\{x \in \mathbb{R}: a<x \leq b\}$, and $] a, b[=\{x \in \mathbb{R}: a<x<b\}$. Throughout this section, let $U$ be a nonempty set. We identify a fuzzy set $\widetilde{a}$ on $U$ with its membership function $\widetilde{a}: U \rightarrow[0,1]$. Let $\mathcal{F}(U)$ be the set of all fuzzy sets on $U$. For $\widetilde{a} \in \mathcal{F}(U)$ and $\alpha \in] 0,1]$, the set

$$
[\widetilde{a}]_{\alpha}=\{x \in U: \widetilde{a}(x) \geq \alpha\}
$$

is called the $\alpha$-level set of $\widetilde{a}$. For a crisp set $S \subset U$, the function $c_{S}: U \rightarrow\{0,1\}$ defined as

$$
c_{S}(x)= \begin{cases}1 & \text { if } x \in S \\ 0 & \text { if } x \notin S\end{cases}
$$

for each $x \in U$ is called the indicator function of $S$. For each $x \in U$, we set $\widetilde{x}=c_{\{x\}} \in \mathcal{F}(U)$. A fuzzy set $\widetilde{a} \in \mathcal{F}(U)$ can be represented as

$$
\widetilde{a}=\sup _{\alpha \in] 0,1]} \alpha c_{[\widetilde{a}]_{\alpha}}
$$

which is well-known as the decomposition theorem; see, for example, [2]. We set

$$
\begin{aligned}
\left.\mathcal{S}(U)=\left\{\left\{S_{\alpha}\right\}_{\alpha \in] 0,1]}: S_{\alpha} \subset U, \alpha \in\right] 0,1\right], & \\
& \text { and } \left.\left.\left.S_{\beta} \supset S_{\gamma} \text { for } \beta, \gamma \in\right] 0,1\right] \text { with } \beta<\gamma\right\},
\end{aligned}
$$

and define $M_{U}: \mathcal{S}(U) \rightarrow \mathcal{F}(U)$ as

$$
M_{U}\left(\left\{S_{\alpha}\right\}_{\alpha \in] 0,1]}\right)=\sup _{\alpha \in] 0,1]} \alpha c_{S_{\alpha}}
$$

for each $\left\{S_{\alpha}\right\}_{\alpha \in] 0,1]} \in \mathcal{S}(U)$. For $\left\{S_{\alpha}\right\}_{\alpha \in] 0,1]} \in \mathcal{S}(U)$ and $x \in U$, it follows that

$$
\left.\left.M_{U}\left(\left\{S_{\alpha}\right\}_{\alpha \in] 0,1]}\right)(x)=\sup _{\alpha \in] 0,1]} \alpha c_{S_{\alpha}}(x)=\sup \{\alpha \in] 0,1\right]: x \in S_{\alpha}\right\}
$$

where $\sup \emptyset=0$. The decomposition theorem can be represented as

$$
\widetilde{a}=M_{U}\left(\left\{[\widetilde{a}]_{\alpha}\right\}_{\alpha \in] 0,1]}\right)
$$

for $\widetilde{a} \in \mathcal{F}(U)$. When $\widetilde{a}=M_{U}\left(\left\{S_{\alpha}\right\}_{\alpha \in] 0,1]}\right)$ for $\widetilde{a} \in \mathcal{F}(U)$ and $\left\{S_{\alpha}\right\}_{\alpha \in] 0,1]} \in \mathcal{S}(U), \widetilde{a}$ is called the fuzzy set generated by $\left\{S_{\alpha}\right\}_{\alpha \in] 0,1]}$, and $\left\{S_{\alpha}\right\}_{\alpha \in] 0,1]}$ is called the generator of $\widetilde{a}$.

The following proposition provides a relationship between a generator of a fuzzy set and level sets of the fuzzy set.

Proposition 2.1. Let $\left\{S_{\alpha}\right\}_{\alpha \in] 0,1]} \in \mathcal{S}(U)$, and let $\widetilde{a}=M_{U}\left(\left\{S_{\alpha}\right\}_{\alpha \in] 0,1]}\right) \in \mathcal{F}(U)$. Then, $[\widetilde{a}]_{\alpha}$ $=\bigcap_{\beta \in] 0, \alpha[} S_{\beta}$ for any $\left.\left.\alpha \in\right] 0,1\right]$.

Proof. Let $\alpha \in] 0,1]$. Then, we have

$$
\begin{aligned}
x \in[\widetilde{a}]_{\alpha} & \left.\Leftrightarrow \widetilde{a}(x)=\sup \{\beta \in] 0,1]: x \in S_{\beta}\right\} \geq \alpha \\
& \left.\Leftrightarrow x \in S_{\beta}, \beta \in\right] 0, \alpha[ \\
& \Leftrightarrow x \in \bigcap_{\beta \in] 0, \alpha[} S_{\beta} .
\end{aligned}
$$


We define orders on $2^{\mathbb{R}}$.

Definition 2.1 ([5, 8-10]). Let $A, B \subset \mathbb{R}$.

(i) We write $A \leq B$ or $B \geq A$ if $B \subset A+\mathbb{R}_{+}$and $A \subset B+\mathbb{R}_{-}$.

(ii) We write $A<B$ or $B>A$ if $B \subset A+\operatorname{int}\left(\mathbb{R}_{+}\right)$and $A \subset B+\operatorname{int}\left(\mathbb{R}_{-}\right)$.

Let $A, B \subset \mathbb{R} . B \subset A+\mathbb{R}_{+}$if and only if for any $v \in B$, there exists $u \in A$ such that $u \leq v . A \subset B+\mathbb{R}_{-}$if and only if for any $u \in A$, there exists $v \in B$ such that $u \leq v$. $B$ $\subset A+\operatorname{int}\left(\mathbb{R}_{+}\right)$if and only if for any $v \in B$, there exists $u \in A$ such that $u<v . A \subset B+$ $\operatorname{int}\left(\mathbb{R}_{-}\right)$if and only if for any $u \in A$, there exists $v \in B$ such that $u<v$.

We define orders on $\mathcal{F}(\mathbb{R})$ based on orderings of level sets of fuzzy sets.

Definition $2.2([5,8,10,12])$. Let $\widetilde{a}, \widetilde{b} \in \mathcal{F}(\mathbb{R})$.

(i) We write $\widetilde{a} \preceq \widetilde{b}$ or $\widetilde{b} \succeq \widetilde{a}$ if $[\widetilde{a}]_{\alpha} \leq[\widetilde{b}]_{\alpha}$ for any $\left.\left.\alpha \in\right] 0,1\right]$.

(ii) We write $\widetilde{a} \prec \widetilde{b}$ or $\widetilde{b} \succ \widetilde{a}$ if $\left.[\widetilde{a}]_{\alpha}<\widetilde{b}\right]_{\alpha}$ for any $\left.\left.\alpha \in\right] 0,1\right]$.

The orders $\preceq$ and $\prec$ in Definition 2.2 are called the fuzzy max order and the strict fuzzy max order on $\mathcal{F}(\mathbb{R})$, respectively. The fuzzy max order for fuzzy numbers has been primarily defined in [12], and many researches have dealt with it. Then, the fuzzy max order for fuzzy numbers has been extended for fuzzy vectors in [10], for fuzzy sets which are closed, convex, normal, and support bounded in [8], and for general fuzzy sets in [5].

\section{Distance and Norm for Crisp Sets}

In this section, a distance and a norm are defined for crisp sets, and their fundamental properties are investigated in order to investigate fundamental properties of a fuzzy distance and a fuzzy norm in the next section.

Throughout the present paper, let $\left(X, d_{X}\right)$ be a metric space, let $\left(Y, d_{Y}\right)$ be a complex vector space equipped with a metric $d_{Y}$ and a zero element $0_{Y}$, and let $(Z,\|\cdot\|)$ be a complex normed space equipped with a zero element $0_{Z}$. Whenever we consider the normed space $(Z,\|\cdot\|)$, a metric $d_{Z}: Z \times Z \rightarrow \mathbb{R}$ is defined as $d_{Z}(x, y)=\|x-y\|$ for each $x, y \in Z$.

A fuzzy set $\widetilde{a} \in \mathcal{F}(X)$ is said to be compact or closed if $[\widetilde{a}]_{\alpha}$ is compact or closed for any $\alpha \in] 0,1]$, respectively. Let $\mathcal{F B C}(X)$ and $\mathcal{F C}(X)$ be sets of all compact and closed fuzzy sets on $X$, respectively.

We define the distance and the norm for crisp sets.

Definition 3.1. (i) For $A, B \subset X, d_{X}(A, B)=\left\{d_{X}(x, y): x \in A, y \in B\right\} \subset \mathbb{R}$ is called the distance between $A$ and $B$.

(ii) For $A \subset Z,\|A\|=\{\|z\|: z \in A\} \subset \mathbb{R}$ is called the norm of $A$.

In Definition 3.1, $d_{X}(A, B)$ is the image of $A \times B$ under the metric $d_{X}: X \times X \rightarrow \mathbb{R}$, and $\|A\|$ is the image of $A$ under the norm $\|\cdot\|: Z \rightarrow \mathbb{R}$.

The following proposition shows properties of the norm for crisp sets.

Proposition 3.1. Let $A, B \subset Z$, and let $\lambda \in \mathbb{C}$.

(i) $A \neq \emptyset \Rightarrow\|A\| \geq\{0\}$.

(ii) $A=\left\{0_{Z}\right\} \Leftrightarrow\|A\|=\{0\}$.

(iii) $\|\lambda A\|=|\lambda|\|A\|$.

(iv) $\|A+B\| \leq\|A\|+\|B\|$. 
Proof. (i) Since $A \neq \emptyset$, it follows that $\|A\| \neq \emptyset$. Since $u \geq 0$ for any $u \in\|A\|$, it follows that $\|A\| \subset\{0\}+\mathbb{R}_{+}$and $\{0\} \subset\|A\|+\mathbb{R}_{-}$. Therefore, we have $\|A\| \geq\{0\}$.

(ii) The necessity is trivial. In order to show the sufficiency, suppose that $A \neq\left\{0_{Z}\right\}$. If $A=\emptyset$, then $\|A\|=\emptyset \neq\{0\}$. Suppose that $A \neq \emptyset$. Then, there exists $z_{0} \in A$ such that $z_{0}$ $\neq 0_{Z}$. Since $0<\left\|z_{0}\right\| \in\|A\|$, we have $\|A\| \neq\{0\}$.

(iii) First, let $u \in\|\lambda A\|$. Then, there exists $x \in A$ such that $u=\|\lambda x\|$. Therefore, we have $u=|\lambda|\|x\| \in|\lambda|\|A\|$. Next, let $v \in|\lambda|\|A\|$. Then, there exists $y \in A$ such that $v=$ $|\lambda|\|y\|$. Therefore, we have $v=\|\lambda y\| \in\|\lambda A\|$.

(iv) First, let $u \in\|A+B\|$. Then, there exist $x \in A$ and $y \in B$ such that $u=\|x+y\|$. Thus, it follows that $u \leq\|x\|+\|y\| \in\|A\|+\|B\|$. Next, let $v \in\|A\|+\|B\|$. Then, there exist $z \in A$ and $w \in B$ such that $v=\|z\|+\|w\|$. Thus, it follows that $v \geq\|z+w\| \in\|A+B\|$. Therefore, we have $\|A+B\| \leq\|A\|+\|B\|$.

The following proposition shows properties of the distance for crisp sets.

Proposition 3.2. Let $A, B, C \subset X$.

(i) $A \neq \emptyset, B \neq \emptyset \Rightarrow d_{X}(A, B) \geq\{0\}$.

(ii) $A=B=\left\{x_{0}\right\}$ for some $x_{0} \in X \Leftrightarrow d_{X}(A, B)=\{0\}$.

(iii) $d_{X}(A, B)=d_{X}(B, A)$.

(iv) $A=\emptyset$ or $C=\emptyset$ or $B=\left\{y_{0}\right\}$ for some $y_{0} \in X \Rightarrow d_{X}(A, C) \leq d_{X}(A, B)+d_{X}(B, C)$.

Proof. (i) Since $A \neq \emptyset$ and $B \neq \emptyset$, it follows that $d_{X}(A, B) \neq \emptyset$. Since $u \geq 0$ for any $u \in$ $d_{X}(A, B)$, it follows that $d_{X}(A, B) \subset\{0\}+\mathbb{R}_{+}$and $\{0\} \subset d_{X}(A, B)+\mathbb{R}_{-}$. Therefore, we have $d_{X}(A, B) \geq\{0\}$.

(ii) The necessity is trivial. In order to show the sufficiency, suppose that $A=B=\left\{x_{0}\right\}$ does not hold for any $x_{0} \in X$. If $A=\emptyset$ or $B=\emptyset$, then $d_{X}(A, B)=\emptyset \neq\{0\}$. Suppose that $A \neq \emptyset$ and $B \neq \emptyset$. Then, it follows that $A \neq\left\{x_{0}\right\}$ or $B \neq\left\{x_{0}\right\}$ for any $x_{0} \in X$. Let $x_{1} \in A$. It follows that $A \neq\left\{x_{1}\right\}$ or $B \neq\left\{x_{1}\right\}$. If $A \neq\left\{x_{1}\right\}$ and $x_{1} \in B$, then $d_{X}(A, B) \neq\{0\}$ since $0<d_{X}\left(x_{2}, x_{1}\right) \in d_{X}(A, B)$ for $x_{2} \in A$ with $x_{2} \neq x_{1}$. If $A \neq\left\{x_{1}\right\}$ and $x_{1} \notin B$, then $d_{X}(A, B) \neq\{0\}$ since $0<d_{X}\left(x_{1}, x_{3}\right) \in d_{X}(A, B)$ for $x_{3} \in B$. If $B \neq\left\{x_{1}\right\}$ and $x_{1} \in B$, then $d_{X}(A, B) \neq\{0\}$ since $0<d_{X}\left(x_{1}, x_{4}\right) \in d_{X}(A, B)$ for $x_{4} \in B$ with $x_{4} \neq x_{1}$. If $B \neq\left\{x_{1}\right\}$ and $x_{1} \notin B$, then $d_{X}(A, B) \neq\{0\}$ since $0<d_{X}\left(x_{1}, x_{5}\right) \in d_{X}(A, B)$ for $x_{5} \in B$.

(iii) We have $d_{X}(A, B)=\left\{d_{X}(x, y): x \in A, y \in B\right\}=\left\{d_{X}(y, x): y \in B, x \in A\right\}=$ $d_{X}(B, A)$.

(iv) If $A=\emptyset$ or $C=\emptyset$, then $d_{X}(A, C)=\emptyset \leq \emptyset=d_{X}(A, B)+d_{X}(B, C)$. Suppose that $A \neq \emptyset, C \neq \emptyset$, and $B=\left\{y_{0}\right\}$ for some $y_{0} \in X$. First, let $u \in d_{X}(A, C)$. Then, there exist $x \in A$ and $z \in C$ such that $u=d_{X}(x, z)$. Thus, it follows that $u \leq d_{X}\left(x, y_{0}\right)+d_{X}\left(y_{0}, z\right) \in$ $d_{X}(A, B)+d_{X}(B, C)$. Next, let $v \in d_{X}(A, B)+d_{X}(B, C)$. Then, there exist $x^{\prime} \in A$ and $z^{\prime}$ $\in C$ such that $v=d_{X}\left(x^{\prime}, y_{0}\right)+d_{X}\left(y_{0}, z^{\prime}\right)$. Thus, it follows that $v \geq d_{X}\left(x^{\prime}, z^{\prime}\right) \in d_{X}(A, C)$. Therefore, we have $d_{X}(A, C) \leq d_{X}(A, B)+d_{X}(B, C)$.

The metric $d_{Y}$ is said to be translation invariant if $d_{Y}(y, z)=d_{Y}(y+x, z+x)$ for any $x, y, z \in Y$, and is said to be homogeneous if $d_{Y}(\lambda x, \lambda y)=|\lambda| d_{Y}(x, y)$ for any $x, y \in Y$ and any $\lambda \in \mathbb{C}$.

The following proposition shows properties of the distance for crisp sets.

Proposition 3.3. Let $A, B, C \subset Y$, and let $\lambda \in \mathbb{C}$.

(i) It does not always hold that $d_{Y}(A, B)=d_{Y}(A+C, B+C)$.

(ii) If $d_{Y}$ is translation invariant, then $d_{Y}(A, B)=d_{Y}(A+x, B+x)$ for any $x \in Y$.

(iii) If $d_{Y}$ is homogeneous, then $d_{Y}(\lambda A, \lambda B)=|\lambda| d_{Y}(A, B)$. 
Proof. (i) We define $d_{Y}: Y \times Y \rightarrow \mathbb{R}$ as

$$
d_{Y}(x, y)= \begin{cases}1 & \text { if } x \neq y \\ 0 & \text { if } x=y\end{cases}
$$

for each $x, y \in Y$. Suppose that $A \neq \emptyset, B \neq \emptyset$, and $A \cap B=\emptyset$. Fix any $x_{0} \in A$ and $y_{0} \in B$, and set $C=\left\{-x_{0},-y_{0}\right\}$. Then, it follows that $d_{Y}(A, B)=\{1\}$. Since $0_{Y} \in(A+C) \cap(B+C)$ from the definition of $C$, it follows that $0=d_{Y}\left(0_{Y}, 0_{Y}\right) \in d_{Y}(A+C, B+C)$. Therefore, we have $d_{Y}(A, B) \neq d_{Y}(A+C, B+C)$.

(ii) Let $x \in Y$. First, let $u \in d_{Y}(A, B)$. Then, there exist $y \in A$ and $z \in B$ such that $u$ $=d_{Y}(y, z)$. Since $d_{Y}$ is translation invariant, we have $u=d_{Y}(y+x, z+x) \in d_{Y}(A+x, B$ $+x)$. Next, let $v \in d_{Y}(A+x, B+x)$. Then, there exist $y^{\prime} \in A$ and $z^{\prime} \in B$ such that $v=$ $d_{Y}\left(y^{\prime}+x, z^{\prime}+x\right)$. Since $d_{Y}$ is translation invariant, we have $v=d_{Y}\left(y^{\prime}, z^{\prime}\right) \in d_{Y}(A, B)$.

(iii) First, let $u \in d_{Y}(\lambda A, \lambda B)$. Then, there exist $x \in A$ and $y \in B$ such that $u=$ $d_{Y}(\lambda x, \lambda y)$. Since $d_{Y}$ is homogeneous, we have $u=|\lambda| d_{Y}(x, y) \in|\lambda| d_{Y}(A, B)$. Next, let $v$ $\in|\lambda| d_{Y}(A, B)$. Then, there exist $x^{\prime} \in A$ and $y^{\prime} \in B$ such that $v=|\lambda| d_{Y}\left(x^{\prime}, y^{\prime}\right)$. Since $d_{Y}$ is homogeneous, we have $v=d_{Y}\left(\lambda x^{\prime}, \lambda y^{\prime}\right) \in d_{Y}(\lambda A, \lambda B)$.

The following proposition provides a relationship between the distance and the norm for crisp sets.

Proposition 3.4. Let $A, B \subset Z$. Then, $d_{Z}(A, B)=\|A-B\|$.

Proof. First, let $u \in d_{Z}(A, B)$. Then, there exist $x \in A$ and $y \in B$ such that $u=d_{Z}(x, y)$. Therefore, we have $u=\|x-y\| \in\|A-B\|$. Next, let $v \in\|A-B\|$. Then, there exist $x^{\prime} \in A$ and $y^{\prime} \in B$ such that $v=\left\|x^{\prime}-y^{\prime}\right\|$. Therefore, we have $v=d_{Z}\left(x^{\prime}, y^{\prime}\right) \in d_{Z}(A, B)$.

\section{Fuzzy Distance and Fuzzy Norm}

In this section, a fuzzy distance and a fuzzy norm are proposed, and their fundamental properties are investigated. Some proofs in this section refer to some auxiliary results in the next section.

We define the fuzzy distance and the fuzzy norm by Zadeh's extension principle. See $[2,6,11,13,17]$ for Zadeh's extension principle.

Definition 4.1. (i) For $\widetilde{a}, \widetilde{b} \in \mathcal{F}(X), d_{X}(\widetilde{a}, \widetilde{b}) \in \mathcal{F}(\mathbb{R})$ defined as

$$
d_{X}(\widetilde{a}, \widetilde{b})(u)=\sup _{u=d_{X}(x, y)} \min \{\widetilde{a}(x), \widetilde{b}(y)\}
$$

for each $u \in \mathbb{R}$ is called the fuzzy distance between $\widetilde{a}$ and $\widetilde{b}$.

(ii) For $\widetilde{a} \in \mathcal{F}(X)$ and $b \in X, d_{X}(\widetilde{a}, b) \in \mathcal{F}(\mathbb{R})$ defined as

$$
d_{X}(\widetilde{a}, b)(u)=\sup _{u=d_{X}(x, b)} \widetilde{a}(x)
$$

for each $u \in \mathbb{R}$ is called the fuzzy distance between $\widetilde{a}$ and $b$. Furthermore, $d_{X}(b, \widetilde{a})=d_{X}(\widetilde{a}, b)$ is called the fuzzy distance between $b$ and $\widetilde{a}$.

(iii) For $\widetilde{a} \in \mathcal{F}(Z),\|\widetilde{a}\| \in \mathcal{F}(\mathbb{R})$ defined as

$$
\|\widetilde{a}\|(u)=\sup _{u=\|x\|} \widetilde{a}(x)
$$

for each $u \in \mathbb{R}$ is called the fuzzy norm of $\widetilde{a}$. 
In Definition 4.1, the fuzzy distance $d_{X}$ is defined for the metric space $X$. Since $Y$ and $Z$ are also metric spaces, fuzzy distances $d_{Y}$ and $d_{Z}$ for $Y$ and $Z$, respectively, are defined similarly.

The following proposition shows a property of the fuzzy distance.

Proposition 4.1. Let $\widetilde{a} \in \mathcal{F}(X)$, and let $b \in X$. Then, $d_{X}(\widetilde{a}, b)=d_{X}\left(\widetilde{a}, c_{\{b\}}\right)$.

Proof. For any $u \in \mathbb{R}$, we have $d_{X}\left(\widetilde{a}, c_{\{b\}}\right)(u)=\sup _{u=d_{X}(x, y)} \min \left\{\widetilde{a}(x), c_{\{b\}}(y)\right\}=\sup _{u=d_{X}(x, b)}$ $\min \left\{\widetilde{a}(x), c_{\{b\}}(b)\right\}=\sup _{u=d_{X}(x, b)} \widetilde{a}(x)=d_{X}(\widetilde{a}, b)(u)$.

The following proposition is a special case of [6, Proposition 2], and provides a relationship between fuzzy norms of fuzzy sets and generators of the fuzzy sets.

Proposition 4.2. Let $\left\{S_{\alpha}\right\}_{\alpha \in] 0,1]} \in \mathcal{S}(Z)$, and let $\widetilde{a}=M_{Z}\left(\left\{S_{\alpha}\right\}_{\alpha \in] 0,1]}\right) \in \mathcal{F}(Z)$. Then, $\|\widetilde{a}\|$ $=M_{\mathbb{R}}\left(\left\{\left\|S_{\alpha}\right\|\right\}_{\alpha \in] 0,1]}\right)=\sup _{\alpha \in] 0,1]} \alpha c_{\left\|S_{\alpha}\right\|}$.

The following proposition is a special case of [6, Proposition 3], and provides a relationship between fuzzy distances of fuzzy sets and generators of the fuzzy sets.

Proposition 4.3. Let $\left\{S_{\alpha}\right\}_{\alpha \in] 0,1]},\left\{T_{\alpha}\right\}_{\alpha \in] 0,1]} \in \mathcal{S}(X)$, and let $\widetilde{a}=M_{X}\left(\left\{S_{\alpha}\right\}_{\alpha \in] 0,1]}\right), \widetilde{b}=$ $M_{X}\left(\left\{T_{\alpha}\right\}_{\alpha \in] 0,1]}\right) \in \mathcal{F}(X)$. Then, $d_{X}(\widetilde{a}, \widetilde{b})=M_{\mathbb{R}}\left(\left\{d_{X}\left(S_{\alpha}, T_{\alpha}\right)\right\}_{\alpha \in] 0,1]}\right)=\sup _{\alpha \in] 0,1]} \alpha c_{d_{X}\left(S_{\alpha}, T_{\alpha}\right)}$.

We define addition, subtraction, and scalar multiplication of fuzzy sets by Zadeh's extension principle.

Definition 4.2. Let $V$ be a complex vector space. For $\widetilde{a}, \widetilde{b} \in \mathcal{F}(V)$ and $\lambda \in \mathbb{C}$, we define $\widetilde{a}$ $+\widetilde{b}, \widetilde{a}-\widetilde{b}, \lambda \widetilde{a} \in \mathcal{F}(V)$ as

$$
\begin{gathered}
(\widetilde{a}+\widetilde{b})(x)=\sup _{x=y+z} \min \{\widetilde{a}(y), \widetilde{b}(z)\} \\
(\widetilde{a}-\widetilde{b})(x)=\sup _{x=y-z} \min \{\widetilde{a}(y), \widetilde{b}(z)\}, \\
(\lambda \widetilde{a})(x)=\sup _{x=\lambda y} \widetilde{a}(y)
\end{gathered}
$$

for each $x \in V$, respectively.

The following proposition is a special case of [6, Propositions 2 and 3], and provides relationships between operations of fuzzy sets and generators of the fuzzy sets.

Proposition 4.4. Let $V$ be a complex vector space, and let $\left\{S_{\alpha}\right\}_{\alpha \in] 0,1]},\left\{T_{\alpha}\right\}_{\alpha \in] 0,1]} \in \mathcal{S}(V)$. In addition, let $\widetilde{a}=M_{V}\left(\left\{S_{\alpha}\right\}_{\alpha \in] 0,1]}\right), \widetilde{b}=M_{V}\left(\left\{T_{\alpha}\right\}_{\alpha \in] 0,1]}\right) \in \mathcal{F}(V)$. Then, $\widetilde{a}+\widetilde{b}=M_{V}\left(\left\{S_{\alpha}+\right.\right.$ $\left.\left.T_{\alpha}\right\}_{\alpha \in] 0,1]}\right)=\sup _{\alpha \in] 0,1]} \alpha c_{S_{\alpha}+T_{\alpha}}, \widetilde{a}-\widetilde{b}=M_{V}\left(\left\{S_{\alpha}-T_{\alpha}\right\}_{\alpha \in] 0,1]}\right)=\sup _{\alpha \in] 0,1]} \alpha c_{S_{\alpha}-T_{\alpha}}$, and $\lambda \widetilde{a}$ $=M_{V}\left(\left\{\lambda S_{\alpha}\right\}_{\alpha \in] 0,1]}\right)=\sup _{\alpha \in] 0,1]} \alpha c_{\lambda S_{\alpha}}$.

The following proposition provides a relationship between addition and subtraction of fuzzy sets.

Proposition 4.5. Let $V$ be a complex vector space, and let $\widetilde{a}, \widetilde{b} \in \mathcal{F}(V)$. Then, $\widetilde{a}+(-1) \widetilde{b}$ $=\widetilde{a}-\widetilde{b}$.

Proof. Since $[\widetilde{a}]_{\alpha}+(-1)[\widetilde{b}]_{\alpha}=[\widetilde{a}]_{\alpha}-[\widetilde{b}]_{\alpha}$ for any $\left.\left.\alpha \in\right] 0,1\right]$, we have $\widetilde{a}+(-1) \widetilde{b}=M_{V}\left(\left\{[\widetilde{a}]_{\alpha}\right.\right.$ $\left.\left.+(-1)[\widetilde{b}]_{\alpha}\right\}_{\alpha \in] 0,1]}\right)=M_{V}\left(\left\{[\widetilde{a}]_{\alpha}-[\widetilde{b}]_{\alpha}\right\}_{\alpha \in] 0,1]}\right)=\widetilde{a}-\widetilde{b}$ from Proposition 4.4 . 
The following proposition provides relationships between the fuzzy distance and the fuzzy norm.

Proposition 4.6. Let $\widetilde{a}, \widetilde{b} \in \mathcal{F}(Z)$.

(i) $d_{Z}(\widetilde{a}, \widetilde{b})=\|\widetilde{a}-\widetilde{b}\|$.

(ii) $d_{Z}\left(\widetilde{a}, \widetilde{0}_{Z}\right)=\left\|\widetilde{a}-\widetilde{0}_{Z}\right\|=d_{Z}\left(\widetilde{a}, 0_{Z}\right)$.

Proof. (i) From the decomposition theorem, it follows that $\widetilde{a}=M_{Z}\left(\left\{[\widetilde{a}]_{\alpha}\right\}_{\alpha \in] 0,1]}\right)$ and $\widetilde{b}=$ $M_{Z}\left(\left\{[\widetilde{b}]_{\alpha}\right\}_{\alpha \in] 0,1]}\right)$. Thus, it follows that $d_{Z}(\widetilde{a}, \widetilde{b})=M_{\mathbb{R}}\left(\left\{d_{Z}\left([\widetilde{a}]_{\alpha},[\widetilde{b}]_{\alpha}\right)\right\}_{\alpha \in] 0,1]}\right)$ from Proposition 4.3. Since $\widetilde{a}-\widetilde{b}=M_{Z}\left(\left\{[\widetilde{a}]_{\alpha}-[\widetilde{b}]_{\alpha}\right\}_{\alpha \in] 0,1]}\right)$ from Proposition 4.4, it follows that $\|\widetilde{a}-\widetilde{b}\|=$ $M_{\mathbb{R}}\left(\left\{\left\|[\widetilde{a}]_{\alpha}-[\widetilde{b}]_{\alpha}\right\|\right\}_{\alpha \in] 0,1]}\right)$ from Proposition 4.2. Since $d_{Z}\left([\widetilde{a}]_{\alpha},[\widetilde{b}]_{\alpha}\right)=\left\|[\widetilde{a}]_{\alpha}-[\widetilde{b}]_{\alpha}\right\|$ for any $\alpha$ $\in] 0,1]$ from Proposition 3.4, we have $d_{Z}(\widetilde{a}, \widetilde{b})=M_{\mathbb{R}}\left(\left\{d_{Z}\left([\widetilde{a}]_{\alpha},[\widetilde{b}]_{\alpha}\right)\right\}_{\alpha \in] 0,1]}\right)=M_{\mathbb{R}}\left(\left\{\|[\widetilde{a}]_{\alpha}-\right.\right.$ $\left.\left.[\widetilde{b}]_{\alpha} \|\right\}_{\alpha \in] 0,1]}\right)=\|\widetilde{a}-\widetilde{b}\|$.

(ii) The conclusion follows from (i) and Proposition 4.1.

The following proposition shows properties of the fuzzy norm.

Proposition 4.7. Let $\widetilde{a}, \widetilde{b} \in \mathcal{F}(Z)$, and let $\lambda \in \mathbb{C}$.

(i) $[\widetilde{a}]_{1} \neq \emptyset \Rightarrow[\|\widetilde{a}\|]_{1} \neq \emptyset \Rightarrow\|\widetilde{a}\| \succeq \widetilde{0}$.

(ii) $\widetilde{a}=\widetilde{0}_{Z} \Leftrightarrow\|\widetilde{a}\|=\widetilde{0}$.

(iii) $\|\lambda \widetilde{a}\|=|\lambda|\|\widetilde{a}\|$.

(iv) If the dimension of $Z$ is finite, and $\widetilde{a}, \widetilde{b} \in \mathcal{F B C}(Z)$, then $\|\widetilde{a}+\widetilde{b}\| \preceq\|\widetilde{a}\|+\|\widetilde{b}\|$.

Proof. (i) First, suppose that $[\widetilde{a}]_{1} \neq \emptyset$. From the decomposition theorem and Proposition 4.2, it follows that $\|\widetilde{a}\|=M_{\mathbb{R}}\left(\left\{\left\|[\widetilde{a}]_{\alpha}\right\|\right\}_{\alpha \in] 0,1]}\right)$. Since $[\widetilde{a}]_{1} \neq \emptyset$, we have $\emptyset \neq\left\|[\widetilde{a}]_{1}\right\| \subset[\|\widetilde{a}\|]_{1}$; see also $\left[11\right.$, Proposition 2.1 (i)]. Next, suppose that $[\|\widetilde{a}\|]_{1} \neq \emptyset$. Then, it follows that $[\|\widetilde{a}\|]_{\alpha} \neq \emptyset$ for any $\left.\left.\alpha \in\right] 0,1\right]$. Fix any $\left.\left.\alpha \in\right] 0,1\right]$. Since $u \geq 0$ for any $u \in[\|\widetilde{a}\|]_{\alpha}$, it follows that $[\|\widetilde{a}\|]_{\alpha} \subset\{0\}+\mathbb{R}_{+}=[\widetilde{0}]_{\alpha}+\mathbb{R}_{+}$and $[\widetilde{0}]_{\alpha}=\{0\} \subset[\|\widetilde{a}\|]_{\alpha}+\mathbb{R}_{-}$, and that $[\|\widetilde{a}\|]_{\alpha} \geq[\widetilde{0}]_{\alpha}$. Therefore, we have $\|\widetilde{a}\| \succeq \widetilde{0}$ by the arbitrariness of $\alpha \in] 0,1]$.

(ii) First, we show the necessity. From the decomposition theorem and Propositions 3.1 (ii) and 4.2, we have $\|\widetilde{a}\|=M_{\mathbb{R}}\left(\left\{\left\|[\widetilde{a}]_{\alpha}\right\|\right\}_{\alpha \in] 0,1]}\right)=M_{\mathbb{R}}\left(\left\{\left\|\left\{0_{Z}\right\}\right\|\right\}_{\alpha \in] 0,1]}\right)=M_{\mathbb{R}}\left(\{\{0\}\}_{\alpha \in] 0,1]}\right)$ $=\widetilde{0}$.

Next, we show the sufficiency. Suppose that $\widetilde{a} \neq \widetilde{0}_{Z}$. Then, there are the following two cases: (ii-1) $\widetilde{a}\left(0_{Z}\right)<1$; (ii-2) $\widetilde{a}\left(0_{Z}\right)=1$, and there exists $x_{0} \in Z$ such that $x_{0} \neq 0_{Z}$ and $\widetilde{a}\left(x_{0}\right)>0$.

(ii-1) We set $\alpha_{0}=\widetilde{a}\left(0_{Z}\right)<1$. For any $\left.\left.\alpha \in\right] \alpha_{0}, 1\right]$, since $0_{Z} \notin[\widetilde{a}]_{\alpha}$, it follows that $0 \notin\left\|[\widetilde{a}]_{\alpha}\right\|$. Since $\left.\left.\|\widetilde{a}\|(0)=M_{\mathbb{R}}\left(\left\{\left\|[\widetilde{a}]_{\alpha}\right\|\right\}_{\alpha \in] 0,1]}\right)(0)=\sup \{\alpha \in] 0,1\right]: 0 \in\left\|[\widetilde{a}]_{\alpha}\right\|\right\} \leq \alpha_{0}<1$ $=\widetilde{0}(0)$ from the decomposition theorem and Proposition 4.2, we have $\|\widetilde{a}\| \neq \widetilde{0}$.

(ii-2) We set $\beta_{0}=\widetilde{a}\left(x_{0}\right)>0$ and $u_{0}=\left\|x_{0}\right\|>0$. For any $\left.\left.\alpha \in\right] 0, \beta_{0}\right]$, since $x_{0} \in[\widetilde{a}]_{\alpha}$, it follows that $u_{0}=\left\|x_{0}\right\| \in\left\|[\widetilde{a}]_{\alpha}\right\|$. Since $\left.\|\widetilde{a}\|\left(u_{0}\right)=M_{\mathbb{R}}\left(\left\{\left\|[\widetilde{a}]_{\alpha}\right\|\right\}_{\alpha \in] 0,1]}\right)\left(u_{0}\right)=\sup \{\alpha \in] 0,1\right]$ : $\left.u_{0} \in\left\|[\widetilde{a}]_{\alpha}\right\|\right\} \geq \beta_{0}>0=\widetilde{0}\left(u_{0}\right)$ from the decomposition theorem and Proposition 4.2, we have $\|\widetilde{a}\| \neq \widetilde{0}$.

(iii) From the decomposition theorem and Propositions 3.1 (iii), 4.2, and 4.4, we have $\|\lambda \widetilde{a}\|=M_{\mathbb{R}}\left(\left\{\left\|\lambda[\widetilde{a}]_{\alpha}\right\|\right\}_{\alpha \in] 0,1]}\right)=M_{\mathbb{R}}\left(\left\{|\lambda|\left\|[\widetilde{a}]_{\alpha}\right\|\right\}_{\alpha \in] 0,1]}\right)=|\lambda|\|\widetilde{a}\|$.

(iv) From Proposition 5.3, it follows that $\widetilde{a}+\widetilde{b} \in \mathcal{F B C}(Z)$. Since $[\|\widetilde{a}\|]_{\alpha}=\left\|[\widetilde{a}]_{\alpha}\right\|$ and $[\|\widetilde{b}\|]_{\alpha}=\left\|[\widetilde{b}]_{\alpha}\right\|$ for any $\left.\left.\alpha \in\right] 0,1\right]$ from Proposition 5.2, it follows that $[\|\widetilde{a}\|]_{\alpha}$ and $[\|\widetilde{b}\|]_{\alpha}$ are compact for any $\alpha \in] 0,1]$, and that $\|\widetilde{a}\|,\|\widetilde{b}\| \in \mathcal{F B C}(\mathbb{R})$. From Propositions 3.1 (iv), 
5.2 , and 5.3, it follows that $[\|\widetilde{a}+\widetilde{b}\|]_{\alpha}=\left\|[\widetilde{a}+\widetilde{b}]_{\alpha}\right\|=\left\|[\widetilde{a}]_{\alpha}+[\widetilde{b}]_{\alpha}\right\| \leq\left\|[\widetilde{a}]_{\alpha}\right\|+\left\|[\widetilde{b}]_{\alpha}\right\|=$ $[\|\widetilde{a}\|]_{\alpha}+[\|\widetilde{b}\|]_{\alpha}=[\|\widetilde{a}\|+\|\widetilde{b}\|]_{\alpha}$ for any $\left.\left.\alpha \in\right] 0,1\right]$. Therefore, we have $\|\widetilde{a}+\widetilde{b}\| \preceq\|\widetilde{a}\|+\|\widetilde{b}\|$.

The following proposition shows properties of the fuzzy distance.

Proposition 4.8. Let $\widetilde{a}, \widetilde{b} \in \mathcal{F}(X)$.

(i) $[\widetilde{a}]_{1} \neq \emptyset,[\widetilde{b}]_{1} \neq \emptyset \Rightarrow\left[d_{X}(\widetilde{a}, \widetilde{b})\right]_{1} \neq \emptyset \Rightarrow d_{X}(\widetilde{a}, \widetilde{b}) \succeq \widetilde{0}$.

(ii) $\widetilde{a}=\widetilde{b}=c_{\left\{x_{0}\right\}}$ for some $x_{0} \in X \Leftrightarrow d_{X}(\widetilde{a}, \widetilde{b})=\widetilde{0}$.

(iii) $d_{X}(\widetilde{a}, \widetilde{b})=d_{X}(\widetilde{b}, \widetilde{a})$.

(iv) Assume that the dimension of $Y$ is finite, and that $d_{Y}$ is translation invariant and homogeneous. Moreover, assume that $\widetilde{a}^{\prime}, \widetilde{c}^{\prime} \in \mathcal{F B C}(Y)$, and that $\widetilde{b}^{\prime}=c_{\{x\}}$ for some $x \in Y$. Then, $d_{Y}\left(\widetilde{a}^{\prime}, \widetilde{c}^{\prime}\right) \preceq d_{Y}\left(\widetilde{a}^{\prime}, \widetilde{b}^{\prime}\right)+d_{Y}\left(\widetilde{b}^{\prime}, \widetilde{c}^{\prime}\right)$.

Proof. (i) First, suppose that $[\widetilde{a}]_{1} \neq \emptyset$ and $[\widetilde{b}]_{1} \neq \emptyset$. From the decomposition theorem and Proposition 4.3, it follows that $d_{X}(\widetilde{a}, \widetilde{b})=M_{\mathbb{R}}\left(\left\{d_{X}\left([\widetilde{a}]_{\alpha},[\widetilde{b}]_{\alpha}\right)\right\}_{\alpha \in] 0,1]}\right)$. Since $[\widetilde{a}]_{1} \neq \emptyset$ and $[\widetilde{b}]_{1}$ $\neq \emptyset$, it follows that $\emptyset \neq d_{X}\left([\widetilde{a}]_{1},[\widetilde{b}]_{1}\right) \subset\left[d_{X}(\widetilde{a}, \widetilde{b})\right]_{1}$; see also [11, Proposition 2.1 (i)]. Next, suppose that $\left[d_{X}(\widetilde{a}, \widetilde{b})\right]_{1} \neq \emptyset$. Then, it follows that $\left[d_{X}(\widetilde{a}, \widetilde{b})\right]_{\alpha} \neq \emptyset$ for any $\left.\left.\alpha \in\right] 0,1\right]$. Fix any $\alpha \in] 0,1]$. Since $u \geq 0$ for any $u \in\left[d_{X}(\widetilde{a}, \widetilde{b})\right]_{\alpha}$, it follows that $\left[d_{X}(\widetilde{a}, \widetilde{b})\right]_{\alpha} \subset\{0\}+\mathbb{R}_{+}$ $=[\widetilde{0}]_{\alpha}+\mathbb{R}_{+}$and $[\widetilde{0}]_{\alpha}=\{0\} \subset\left[d_{X}(\widetilde{a}, \widetilde{b})\right]_{\alpha}+\mathbb{R}_{-}$, and that $\left[d_{X}(\widetilde{a}, \widetilde{b})\right]_{\alpha} \geq[\widetilde{0}]_{\alpha}$. Therefore, we have $d_{X}(\widetilde{a}, \widetilde{b}) \succeq \widetilde{0}$ by the arbitrariness of $\left.\left.\alpha \in\right] 0,1\right]$.

(ii) First, we show the necessity. From the decomposition theorem and Propositions 3.2 (ii) and 4.3, we have $d_{X}(\widetilde{a}, \widetilde{b})=M_{\mathbb{R}}\left(\left\{d_{X}\left([\widetilde{a}]_{\alpha},[\widetilde{b}]_{\alpha}\right)\right\}_{\alpha \in] 0,1]}\right)=M_{\mathbb{R}}\left(\left\{d_{X}\left(\left\{x_{0}\right\},\left\{x_{0}\right\}\right)\right\}_{\alpha \in] 0,1]}\right)=$ $M_{\mathbb{R}}\left(\{\{0\}\}_{\alpha \in] 0,1]}\right)=\widetilde{0}$.

Next, we show the sufficiency. From the decomposition theorem and Proposition 4.3, it follows that

$$
d_{X}(\widetilde{a}, \widetilde{b})=M_{\mathbb{R}}\left(\left\{d_{X}\left([\widetilde{a}]_{\alpha},[\widetilde{b}]_{\alpha}\right)\right\}_{\alpha \in] 0,1]}\right)=\widetilde{0} .
$$

In order to show that $[\widetilde{a}]_{\alpha} \cap[\widetilde{b}]_{\alpha} \neq \emptyset$ for any $\left.\alpha \in\right] 0,1\left[\right.$, suppose that there exists $\left.\alpha_{0} \in\right] 0,1[$ such that $[\widetilde{a}]_{\alpha_{0}} \cap[\widetilde{b}]_{\alpha_{0}}=\emptyset$. For any $\left.\left.\alpha \in\right] \alpha_{0}, 1\right]$, since $[\widetilde{a}]_{\alpha} \cap[\widetilde{b}]_{\alpha}=\emptyset$, it follows that $0 \notin$ $d_{X}\left([\widetilde{a}]_{\alpha},[\widetilde{b}]_{\alpha}\right)$. Then, it follows that $\left.\left.d_{X}(\widetilde{a}, \widetilde{b})(0)=\sup \{\alpha \in] 0,1\right]: 0 \in d_{X}\left([\widetilde{a}]_{\alpha},[\widetilde{b}]_{\alpha}\right)\right\} \leq \alpha_{0}<$ $1=\widetilde{0}(0)$, which contradicts (4.1). Fix any $\left.\alpha_{1} \in\right] 0,1\left[\right.$, and let $x_{0} \in[\widetilde{a}]_{\alpha_{1}} \cap[\widetilde{b}]_{\alpha_{1}}$. In order to show that

$$
[\widetilde{a}]_{\alpha_{1}}=[\widetilde{b}]_{\alpha_{1}}=\left\{x_{0}\right\}
$$

suppose that $[\widetilde{a}]_{\alpha_{1}} \neq\left\{x_{0}\right\}$ or $[\widetilde{b}]_{\alpha_{1}} \neq\left\{x_{0}\right\}$. We show only the case $[\widetilde{a}]_{\alpha_{1}} \neq\left\{x_{0}\right\}$. It can be shown the other case $[\widetilde{b}]_{\alpha_{1}} \neq\left\{x_{0}\right\}$ similarly. Then, there exists $x_{1} \in[\widetilde{a}]_{\alpha_{1}}$ such that $x_{1}$ $\neq x_{0}$. Since $x_{0} \in[\widetilde{b}]_{\alpha_{1}}$, it follows that $0<d_{X}\left(x_{1}, x_{0}\right) \in d_{X}\left([\widetilde{a}]_{\alpha_{1}},[\widetilde{b}]_{\alpha_{1}}\right)$. Since $d_{X}\left(x_{1}, x_{0}\right)$ $\in d_{X}\left([\widetilde{a}]_{\alpha},[\widetilde{b}]_{\alpha}\right)$ for any $\left.\left.\alpha \in\right] 0, \alpha_{1}\right]$, it follows that $\left.d_{X}(\widetilde{a}, \widetilde{b})\left(d_{X}\left(x_{1}, x_{0}\right)\right)=\sup \{\alpha \in] 0,1\right]$ : $\left.\left.d_{X}\left(x_{1}, x_{0}\right) \in d_{X}\left([\widetilde{a}]_{\alpha}, \widetilde{[b}\right]_{\alpha}\right)\right\} \geq \alpha_{1}>0=\widetilde{0}\left(d_{X}\left(x_{1}, x_{0}\right)\right)$, which contradicts (4.1). Now, we show that

$$
\left.[\widetilde{a}]_{\alpha}=[\widetilde{b}]_{\alpha}=\left\{x_{0}\right\} \text { for any } \alpha \in\right] 0,1[.
$$

From the same arguments of the proof of (4.2), it follows that $[\widetilde{a}]_{\alpha}=[\widetilde{b}]_{\alpha}$ is a singleton for each $\alpha \in] 0,1\left[\right.$. Suppose that there exist $\left.\alpha_{2} \in\right] 0,1\left[\right.$ and $x_{2} \in X$ such that $[\widetilde{a}]_{\alpha_{2}}=[\widetilde{b}]_{\alpha_{2}}$ $=\left\{x_{2}\right\}$ and $x_{2} \neq x_{0}$. We show only the case $\alpha_{1}<\alpha_{2}$. It can be shown the other case $\alpha_{1}$ $>\alpha_{2}$ similarly. Then, it follows that $x_{2} \in[\widetilde{a}]_{\alpha_{2}} \cap[\widetilde{b}]_{\alpha_{2}} \subset[\widetilde{a}]_{\alpha_{1}} \cap[\widetilde{b}]_{\alpha_{1}}$ and $x_{2} \neq x_{0}$, which 
contradicts (4.2). From (4.3), the decomposition theorem, and Proposition 2.1, it follows that

$$
[\widetilde{a}]_{1}=\bigcap_{\alpha \in] 0,1[}[\widetilde{a}]_{\alpha}=\left\{x_{0}\right\}, \quad[\widetilde{b}]_{1}=\bigcap_{\alpha \in] 0,1[}[\widetilde{b}]_{\alpha}=\left\{x_{0}\right\} .
$$

Therefore, we have $\widetilde{a}=\widetilde{b}=c_{\left\{x_{0}\right\}}$ from (4.3) and (4.4).

(iii) From the decomposition theorem and Propositions 3.2 (iii) and 4.3, we have $d_{X}(\widetilde{a}, \widetilde{b})$ $=M_{\mathbb{R}}\left(\left\{d_{X}\left([\widetilde{a}]_{\alpha},[\widetilde{b}]_{\alpha}\right)\right\}_{\alpha \in] 0,1]}\right)=M_{\mathbb{R}}\left(\left\{d_{X}\left([\widetilde{b}]_{\alpha},[\widetilde{a}]_{\alpha}\right)\right\}_{\alpha \in] 0,1]}\right)=d_{X}(\widetilde{b}, \widetilde{a})$.

(iv) Define $\|\cdot\|_{Y}: Y \rightarrow \mathbb{R}$ as $\left\|x^{\prime}\right\|_{Y}=d_{Y}\left(x^{\prime}, 0_{Y}\right)$ for each $x^{\prime} \in Y$. Then, we have $d_{Y}\left(\widetilde{a}^{\prime}\right.$, $\widetilde{c})=\left\|\widetilde{a}^{\prime}-\widetilde{c}\right\|_{Y}=\left\|\left(\widetilde{a}^{\prime}-\widetilde{b}^{\prime}\right)+\left(\widetilde{b^{\prime}}-\widetilde{c}^{\prime}\right)\right\|_{Y} \preceq\left\|\widetilde{a}^{\prime}-\widetilde{b}^{\prime}\right\|_{Y}+\left\|\widetilde{b^{\prime}}-\widetilde{c}^{\prime}\right\|_{Y}=d_{Y}\left(\widetilde{a}^{\prime}, \widetilde{b}^{\prime}\right)+d_{Y}\left(\widetilde{b^{\prime}}, \widetilde{c}^{\prime}\right)$ from Propositions 4.4, 4.6, 4.7 (iv), and 5.4.

The following proposition shows properties of the fuzzy distance.

Proposition 4.9. Let $\widetilde{a}, \widetilde{b}, \widetilde{c} \in \mathcal{F}(Y)$, and let $\lambda \in \mathbb{C}$.

(i) It does not always hold that $d_{Y}(\widetilde{a}, \widetilde{b})=d_{Y}(\widetilde{a}+\widetilde{c}, \widetilde{b}+\widetilde{c})$.

(ii) If $d_{Y}$ is translation invariant, then $d_{Y}(\widetilde{a}, \widetilde{b})=d_{Y}\left(\widetilde{a}+c_{\{x\}}, \widetilde{b}+c_{\{x\}}\right)$ for any $x \in Y$.

(iii) If $d_{Y}$ is homogeneous, then $d_{Y}(\lambda \widetilde{a}, \lambda \widetilde{b})=|\lambda| d_{Y}(\widetilde{a}, \widetilde{b})$.

Proof. (i) Let $d_{Y}$ and $A, B, C \subset Y$ be the same ones as in the proof of Proposition 3.3 (i). Then, it follows that $d_{Y}(A, B) \neq d_{Y}(A+C, B+C)$. We set $\widetilde{a}=c_{A}, \widetilde{b}=c_{B}$, and $\widetilde{c}$ $=c_{C}$. From the decomposition theorem and Propositions 4.3 and 4.4 , we have $d_{Y}(\widetilde{a}, \widetilde{b})=$ $M_{\mathbb{R}}\left(\left\{d_{Y}\left([\widetilde{a}]_{\alpha},[\widetilde{b}]_{\alpha}\right)\right\}_{\alpha \in] 0,1]}\right)=M_{\mathbb{R}}\left(\left\{d_{Y}(A, B)\right\}_{\alpha \in] 0,1]}\right)=c_{d_{Y}(A, B)} \neq c_{d_{Y}(A+C, B+C)}=M_{\mathbb{R}}\left(\left\{d_{Y}(\right.\right.$ $\left.A+C, B+C)\}_{\alpha \in] 0,1]}\right)=M_{\mathbb{R}}\left(\left\{d_{Y}\left([\widetilde{a}]_{\alpha}+[\widetilde{c}]_{\alpha},[\widetilde{b}]_{\alpha}+[\widetilde{c}]_{\alpha}\right)\right\}_{\alpha \in] 0,1]}\right)=d_{Y}(\widetilde{a}+\widetilde{c}, \widetilde{b}+\widetilde{c})$.

(ii) Let $x \in Y$. From the decomposition theorem and Propositions 3.3 (ii), 4.3, and 4.4, we have $d_{Y}(\widetilde{a}, \widetilde{b})=M_{\mathbb{R}}\left(\left\{d_{Y}\left([\widetilde{a}]_{\alpha},[\widetilde{b}]_{\alpha}\right)\right\}_{\alpha \in] 0,1]}\right)=M_{\mathbb{R}}\left(\left\{d_{Y}\left([\widetilde{a}]_{\alpha}+x,[\widetilde{b}]_{\alpha}+x\right)\right\}_{\alpha \in] 0,1]}\right)=M_{\mathbb{R}}($ $\left.\left\{d_{Y}\left([\widetilde{a}]_{\alpha}+\left[c_{\{x\}}\right]_{\alpha},[\widetilde{b}]_{\alpha}+\left[c_{\{x\}}\right]_{\alpha}\right)\right\}_{\alpha \in] 0,1]}\right)=d_{Y}\left(\widetilde{a}+c_{\{x\}}, \widetilde{b}+c_{\{x\}}\right)$.

(iii) From the decomposition theorem and Propositions 3.3 (iii), 4.3, and 4.4, we have $d_{Y}(\lambda \widetilde{a}, \lambda \widetilde{b})=M_{\mathbb{R}}\left(\left\{d_{Y}\left(\lambda[\widetilde{a}]_{\alpha}, \lambda[\widetilde{b}]_{\alpha}\right)\right\}_{\alpha \in] 0,1]}\right)=M_{\mathbb{R}}\left(\left\{|\lambda| d_{Y}\left([\widetilde{a}]_{\alpha},[\widetilde{b}]_{\alpha}\right)\right\}_{\alpha \in] 0,1]}\right)=|\lambda| d_{Y}(\widetilde{a}, \widetilde{b})$.

\section{Auxiliary Results}

In this section, some auxiliary results referred to in the previous section are presented.

The following proposition gives conditions such that for a mapping, level sets of a fuzzy set obtained from two other fuzzy sets by Zadeh's extension principle coincide with images of level sets of the two fuzzy sets under the mapping.

Proposition 5.1. Let $\left(X_{i}, d_{i}\right), i=1,2,3$ be metric spaces. If $f: X_{1} \times X_{2} \rightarrow X_{3}$ is continuous, then $\left[f\left(\widetilde{a}_{1}, \widetilde{a}_{2}\right)\right]_{\alpha}=f\left(\left[\widetilde{a}_{1}\right]_{\alpha},\left[\widetilde{a}_{2}\right]_{\alpha}\right)$ for any $\left.\left.\alpha \in\right] 0,1\right]$ and any $\widetilde{a}_{i} \in \mathcal{F B C}\left(X_{i}\right), i=1,2$, where $f\left(\widetilde{a}_{1}, \widetilde{a}_{2}\right) \in \mathcal{F}\left(X_{3}\right)$ is defined as $f\left(\widetilde{a}_{1}, \widetilde{a}_{2}\right)\left(x_{3}\right)=\sup _{x_{3}=f\left(x_{1}, x_{2}\right)} \min \left\{\widetilde{a}_{1}\left(x_{1}\right), \widetilde{a}_{2}\left(x_{2}\right)\right\}$ for each $x_{3} \in X_{3}$, and a metric on $X_{1} \times X_{2}$ is the product metric defined as $d_{\pi}\left(\left(x_{1}, x_{2}\right),\left(y_{1}, y_{2}\right)\right)$ $=\max \left\{d_{1}\left(x_{1}, y_{1}\right), d_{2}\left(x_{2}, y_{2}\right)\right\}$ for each $\left(x_{1}, x_{2}\right),\left(y_{1}, y_{2}\right) \in X_{1} \times X_{2}$. See also [3, Definition 10.11] for the product metric.

Proof. It is sufficient to show that $f^{-1}\left(x_{3}\right)=\emptyset$ or $\sup _{\left(x_{1}, x_{2}\right) \in f^{-1}\left(x_{3}\right)} \min \left\{\widetilde{a}_{1}\left(x_{1}\right), \widetilde{a}_{2}\left(x_{2}\right)\right\}$ is attained for any $x_{3} \in X_{3}$ from [11, Proposition 3.3]. In order to show it, let $\widehat{x}_{3} \in X_{3}$, let $f^{-1}\left(\widehat{x}_{3}\right) \neq \emptyset$, and we show that $\sup _{\left(x_{1}, x_{2}\right) \in f^{-1}\left(\widehat{x}_{3}\right)} \min \left\{\widetilde{a}_{1}\left(x_{1}\right), \widetilde{a}_{2}\left(x_{2}\right)\right\}$ is attained. Note that $\widetilde{a}_{i}$ $\in \mathcal{F B C}\left(X_{i}\right), i=1,2$ are upper semicontinuous. Let $\widetilde{b} \in \mathcal{F}\left(X_{1} \times X_{2}\right)$ be a fuzzy set defined as $\widetilde{b}\left(x_{1}, x_{2}\right)=\min \left\{\widetilde{a}_{1}\left(x_{1}\right), \widetilde{a}_{2}\left(x_{2}\right)\right\}$ for each $\left(x_{1}, x_{2}\right) \in X_{1} \times X_{2}$. The product metric $d_{\pi}$ defines 
the product topology on $X_{1} \times X_{2}$. Since $[\widetilde{b}]_{\alpha}=\left[\widetilde{a}_{1}\right]_{\alpha} \times\left[\widetilde{a}_{2}\right]_{\alpha}$ is compact for each $\left.\left.\alpha \in\right] 0,1\right]$ from Tihonov's theorem, it follows that $\widetilde{b}$ is upper semicontinuous. If $\sup _{\left(x_{1}, x_{2}\right) \in f^{-1}\left(\widehat{x}_{3}\right)} \widetilde{b}\left(x_{1}, x_{2}\right)$ $=0$, then $\sup _{\left(x_{1}, x_{2}\right) \in f^{-1}\left(\widehat{x}_{3}\right)} \widetilde{b}\left(x_{1}, x_{2}\right)$ is attained. Suppose that $\sup _{\left(x_{1}, x_{2}\right) \in f^{-1}\left(\widehat{x}_{3}\right)} \widetilde{b}\left(x_{1}, x_{2}\right)>0$. Then, there exists $\left(\widehat{x}_{1}, \widehat{x}_{2}\right) \in f^{-1}\left(\widehat{x}_{3}\right)$ such that $\widetilde{b}\left(\widehat{x}_{1}, \widehat{x}_{2}\right)>0$. Let $\left.\left.\widehat{\alpha} \in\right] 0, \widetilde{b}\left(\widehat{x}_{1}, \widehat{x}_{2}\right)\right]$. It follows that $\left(\widehat{x}_{1}, \widehat{x}_{2}\right) \in\left[\widetilde{b}_{\widehat{\alpha}}=\left[\widetilde{a}_{1}\right]_{\widehat{\alpha}} \times\left[\widetilde{a}_{2}\right]_{\widehat{\alpha}}\right.$. Since $f^{-1}\left(\widehat{x}_{3}\right)$ is closed from the continuity of $f$, and $[\widetilde{b}]_{\widehat{\alpha}}$ is compact, it follows that $f^{-1}\left(\widehat{x}_{3}\right) \cap[\widetilde{b}]_{\widehat{\alpha}}$ is compact. Since $\sup _{\left(x_{1}, x_{2}\right) \in f^{-1}\left(\widehat{x}_{3}\right)} \widetilde{b}\left(x_{1}, x_{2}\right)$ $=\sup _{\left(x_{1}, x_{2}\right) \in f^{-1}\left(\widehat{x}_{3}\right) \cap[\widetilde{b}]_{\widehat{\alpha}}} \widetilde{b}\left(x_{1}, x_{2}\right), f^{-1}\left(\widehat{x}_{3}\right) \cap[\widetilde{b}]_{\widehat{\alpha}}$ is a nonempty compact set, and $\widetilde{b}$ is upper semicontinuous, it follows that $\sup _{\left(x_{1}, x_{2}\right) \in f^{-1}\left(\widehat{x}_{3}\right)} \widetilde{b}\left(x_{1}, x_{2}\right)$ is attained.

The following proposition gives conditions such that level sets of the fuzzy norm of a fuzzy set coincide with images of level sets of the fuzzy set under the norm.

Proposition 5.2. Assume that the dimension of $Z$ is finite. Let $\widetilde{a} \in \mathcal{F C}(Z)$. Then, $[\|\widetilde{a}\|]_{\alpha}$ $=\left\|[\widetilde{a}]_{\alpha}\right\|$ for any $\left.\left.\alpha \in\right] 0,1\right]$.

Proof. Define $f: Z \rightarrow \mathbb{R}$ as $f(x)=\|x\|$ for each $x \in Z$. It is sufficient to show that $f^{-1}(\lambda)$ $=\emptyset$ or $\sup _{x \in f^{-1}(\lambda)} \widetilde{a}(x)$ is attained for any $\lambda \in \mathbb{R}$ from [11, Proposition 3.3]. In order to show it, let $\widehat{\lambda} \in \mathbb{R}$, let $f^{-1}(\widehat{\lambda}) \neq \emptyset$, and we show that $\sup _{x \in f^{-1}(\widehat{\lambda})} \widetilde{a}(x)$ is attained. Since $f^{-1}(\widehat{\lambda}) \neq \emptyset$, it follows that $\widehat{\lambda} \geq 0$. Since $f^{-1}(\widehat{\lambda})$ is compact, and $\widetilde{a}$ is upper semicontinuous, it follows that $\sup _{x \in f^{-1}(\widehat{\lambda})} \widetilde{a}(x)$ is attained.

The following proposition gives conditions such that level sets of addition of two fuzzy sets coincide with additions of level sets of the two fuzzy sets.

Proposition 5.3. Assume that the dimension of $Z$ is finite. Let $\widetilde{a}, \widetilde{b} \in \mathcal{F B C}(Z)$. Then, $[\widetilde{a}$ $+\widetilde{b}]_{\alpha}=[\widetilde{a}]_{\alpha}+[\widetilde{b}]_{\alpha}$ for any $\left.\left.\alpha \in\right] 0,1\right]$.

Proof. We define $f: Z \times Z \rightarrow Z$ as $f(x, y)=x+y$ for each $(x, y) \in Z \times Z$. Since $f$ is a linear mapping, $f$ is continuous (with respect to any norm on $Z \times Z$ ). Therefore, we have the conclusion from Proposition 5.1.

The following proposition gives conditions such that a norm is induced by a metric, and shows a relationship between the metric and the induced norm.

Proposition 5.4. Assume that $d_{Y}$ is translation invariant and homogeneous. Define $\|\cdot\|_{Y}$ : $Y \rightarrow \mathbb{R}$ as $\|x\|_{Y}=d_{Y}\left(x, 0_{Y}\right)$ for each $x \in Y$. Then, $\|\cdot\|_{Y}$ is a norm on $Y$. Furthermore, $d_{Y}(x, y)=\|x-y\|_{Y}$ for any $x, y \in Y$.

Proof. Since $d_{Y}$ is a metric on $Y$, for any $x \in Y$, it follows that $\|x\|_{Y}=d_{Y}\left(x, 0_{Y}\right) \geq 0$, and that $\|x\|_{Y}=d_{Y}\left(x, 0_{Y}\right)=0$ if and only if $x=0_{Y}$. Since $d_{Y}$ is homogeneous, it follows that $\|\lambda x\|_{Y}=d_{Y}\left(\lambda x, 0_{Y}\right)=d_{Y}\left(\lambda x, \lambda 0_{Y}\right)=|\lambda| d_{Y}\left(x, 0_{Y}\right)=|\lambda|\|x\|_{Y}$ for any $x \in Y$ and any $\lambda \in \mathbb{C}$. Since $d_{Y}$ is translation invariant, it follows that $\|x+y\|_{Y}=d_{Y}\left(x+y, 0_{Y}\right)=d_{Y}(x,-y) \leq$ $d_{Y}\left(x, 0_{Y}\right)+d_{Y}\left(0_{Y},-y\right)=\|x\|_{Y}+d_{Y}\left(y, 0_{Y}\right)=\|x\|_{Y}+\|y\|_{Y}$ for any $x, y \in Y$. Therefore, $\|\cdot\|_{Y}$ is a norm on $Y$. Since $d_{Y}$ is translation invariant, we have $d_{Y}(x, y)=d_{Y}\left(x-y, 0_{Y}\right)$ $=\|x-y\|_{Y}$ for any $x, y \in Y$. 


\section{Conclusions}

In the present paper, a fuzzy distance and a fuzzy norm were proposed. The fuzzy distance and the fuzzy norm were defined by Zadeh's extension principle, and their fundamental properties were investigated. Fuzzy distances and fuzzy norms can be expected to be useful for measuring the difference of data involving uncertainty or vagueness.

Given data which represent characteristics of individuals, the difference of characteristics of any two individuals is usually measured by a distance or a norm of some kind, and the data are analyzed based on the measured differences. However, such data sometimes involve uncertainty or vagueness caused by observations or human decisions. In this case, it is suitable to represent characteristics of individuals as sets rather than points. Furthermore, it is natural that such sets have uncertain or vague boundaries. Therefore, it is more suitable to represent characteristics of individuals as fuzzy sets rather than sets. Fuzzy distances and fuzzy norms are needed and important to analyze such data represented as fuzzy sets.

Mathematical programming problems often involve crisp distances or norms. When fuzzy distances or fuzzy norms are considered instead of the crisp distances or norms, the problems are fuzzy mathematical programming problems. For example, a crisp minisum location problem is a problem to find the location of a facility which minimizes the total sum of crisp distances between the facility and demand points. Demand points are fixed points which represent locations of customers of the facility to be located. If demand points in the minisum location problem are considered as fuzzy sets, then the problem is a fuzzy minisum location problem involving fuzzy distances. In this case, the fuzzy sets represent uncertain or vague locations of demands. The obtained results in the present paper can be expected to be useful for analyzing various fuzzy mathematical programming problems such as the fuzzy minisum location problem.

\section{References}

[1] D. Diamond and P. Kloeden: Metric Spaces of Fuzzy Sets (World Scientific, 1994).

[2] D. Dubois, W. Ostasiewicz, and H. Prade: Fuzzy sets: history and basic notions. In D. Dubois and H. Prade (eds.): Fundamentals of Fuzzy Sets (Kluwer Academic Publishers, Boston, MA, 2000), 21-124.

[3] J.R. Giles: Introduction to the Analysis of Normed Linear Spaces (Cambridge, 2000).

[4] S.K. Gupta and D. Dangar: Duality for a class of fuzzy nonlinear optimization problem under generalized convexity. Fuzzy Optimization and Decision Making, 13 (2014), 131150.

[5] M. Kon: Operation and ordering of fuzzy sets, and fuzzy set-valued convex mappings. Journal of Fuzzy Set Valued Analysis (2014), Article ID jfsva-00202, 17 pages.

[6] M. Kon: A note on Zadeh's extension principle. Applied and Computational Mathematics, 4 (2015), 10-14.

[7] M. Kurano, M. Yasuda, J. Nakagami, and Y. Yoshida: Markov-type fuzzy decision processes with discounted reward on a closed interval. European Journal of Operational Research, 92 (1996), 649-662.

[8] M. Kurano, M. Yasuda, J. Nakagami, and Y. Yoshida: Ordering of convex fuzzy sets - a brief survey and new results. Journal of the Operations Research Society of Japan, 43 (2000), 138-148.

[9] D. Kuroiwa, T. Tanaka, and T.X.D. Ha: On cone convexity of set-valued maps. Nonlinear Analysis, 30 (1997), 1487-1496. 
[10] T. Maeda: On characterization of fuzzy vectors and its applications to fuzzy mathematical programming problems. Fuzzy Sets and Systems, 159 (2008), 3333-3346.

[11] H.T. Nguyen: A note on the extension principle for fuzzy sets. Journal of Mathematical Analysis and Applications, 64 (1978), 369-380.

[12] J. Ramík and J. Řimánek: Inequality relation between fuzzy numbers and its use in fuzzy optimization. Fuzzy Sets and Systems, 16 (1985), 123-138.

[13] H.-C. Wu: Duality theory in fuzzy optimization problems formulated by the Wolfe's primal and dual pair. Fuzzy Optimization and Decision Making, 6 (2007), 179-198.

[14] H.-C. Wu: The optimality conditions for optimization problems with fuzzy-valued objective functions. Optimization, 57 (2008), 473-489.

[15] H.-C. Wu: The optimality conditions for optimization problems with convex constraints and multiple fuzzy-valued objective functions. Fuzzy Optimization and Decision Making, 8 (2009), 295-321.

[16] Y. Yoshida: A time-average fuzzy reward criterion in fuzzy decision processes. Information Sciences, 110 (1998), 103-112.

[17] L.A. Zadeh: Fuzzy sets. Information and Control, 8 (1965), 338-353.

Masamichi Kon

Faculty of Science and Technology

Hirosaki University

3 Bunkyo, Hirosaki

Aomori 036-8561, Japan

E-mail: masakon@hirosaki-u.ac.jp 